Ann. Zootech., 1978, 27 (4), 639-645.

\title{
Note \\ Estimation du poids du contenu digestif des bovins à partir du poids du contenu du rumen
}

\author{
C. BERANGER et J. ROBEIIN \\ Laboratoive de la Production de Viande, \\ Centre de Recherches de Clermont-Ferrand, I.N.R.A., \\ Theix, Saint-Genès-Champanelle, 631 to Braumont (France)
}

\section{Résumé}

D'après les mesures effectuées à l'abattage sur ı 98 bovins, on a estimé le poids du contenu digestif total à partir du poids du contenu du réticulo-rumen. La précision de cette estimation est de $5 \mathrm{p}$. roo, et cela permet de déterniner le poids vif vide des bovins à I p. roo près, connaissant le poids vif, le poids du contenu du rumen, et éventuellenient la nature ou la digestibilité du réginte alinentaire consommé avant l'abattage.

Le poids du contenu digestif des bovins représente en movenne i5 p. Ioo du poids vif de l'animal et varie de façon considérable (de 5 à $25 \mathrm{p}$. I 100 du poids vif) en fonction de nombreux facteurs, en particulier du régime alimentaire, de la quantité ingérée et du temps écoulé depuis le repas. Sa détermination est nécessaire pour connaître la masse corporelle réelle de l'animal que le poids vif ne reflète que grossièrement compte tenu de ces variations du contenu digestif. A l'abattage, il est indispensable de peser le contenu digestif (tractus digestif plein-tractus digestif vide) pour connaître le poids vif vide, c'est-à-dire la masse corporelle et déterminer le rendement vrai en carcasse $\frac{\text { Ioo } \times \text { Poids de carcasse; }}{\text { Poids vif vide }}$ ce dernier est le seul rendement caractéristique de la valeur bouchère de l'animal, le rendement commercial roo $\times \frac{\text { Poids de carcasse }}{\text { Poids vif }}$ étant tributaire des variations du poids du contenu digestif et donc des conditions d'alimentation et d'abattage (jeûne, transport...). 


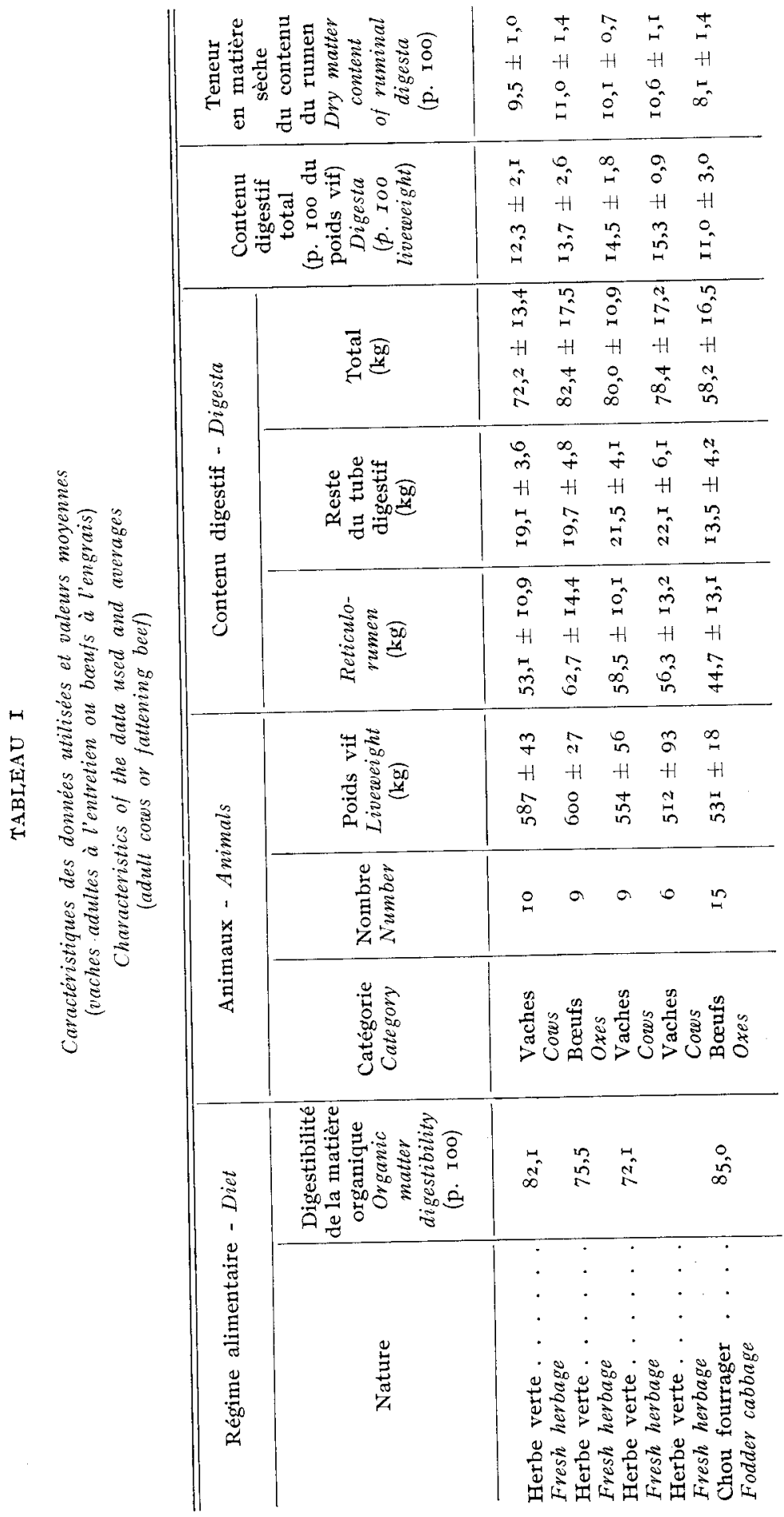




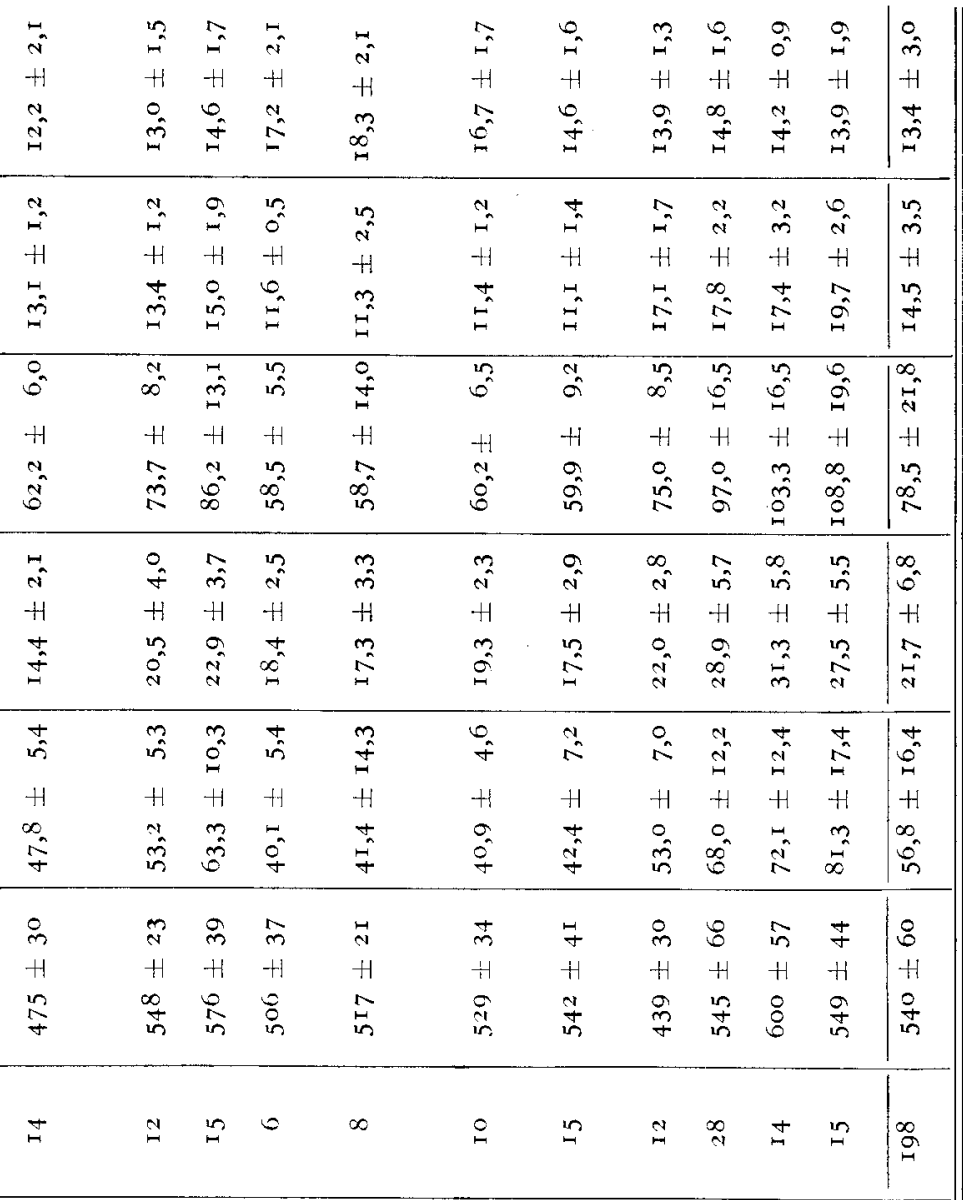

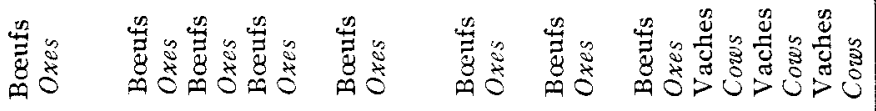

$\begin{array}{lll}n & 0 & 2 \\ \infty & 0 & 0\end{array}$

î $8^{n}$ in in in

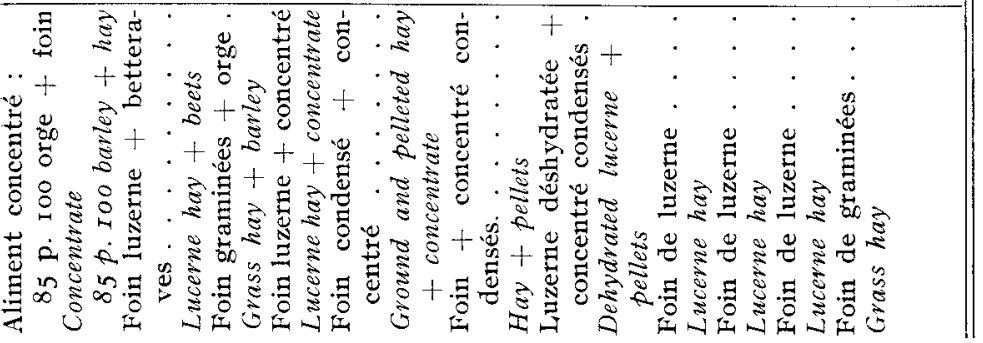


Cette mesure du poids du contenu digestif est très difficile à réaliser dans un abattoir commercial sans perturber considérablement le travail; la vidange du feuillet et des intestins est longue et donc coûteuse. C'est pourquoi nous avons cherché à estimer le poids du contenu du tube digestif à partir du seul contenu du réticulo-rumen relativement facile et rapide à mesurer. Le contenu du rumen constitue en effet en moyenne 72 p. Ioo $(67$ à 77$)$ du contenu digestif total et varie davantage que le contenu du reste du tube digestif sous l'influence des divers facteurs. En outre, le rumen pent être vidé et son contenu pesé sur l'animal vivant porteur d'une large canule.

\section{Origine des données}

Nous avons mesuré les poids du contenu du rumen et du contenu digestif total de $\mathrm{I} 98$ boufs et vaches, ayant reçu I 6 régimes alimentaires différents, contrôlés et ayant été abattus $2,5-3$ heures ou $4,5-5$ heures ou $8,5-9$ heures out I3 heures après le début du repas, dans le cadre d'une série d'expériences planifiées pour étudier la cinétique de la digestion chez les bovins. Les animaux avaient sans cesse de l'eau à leur disposition jusqu'à l'abattage; ils étaient pesés aussitôt avant l'abattage et les différentes parties du tube digestif pesées pleines et vides aussitôt après. Le rumen était ligaturé au niveau de 1'orifice réticulo-omasa1; le contenu du rectum était compris dans le contenu total. Nous connaissions la digestibilité de la matière organique de i2 rations alimentaires et la teneur en matière sèche du contenu de rumen de tous les animaux (tabl. I).

\section{Résultats}

On a étudié les variations du poids du contenu du reste du tube digestif par analyse de covariance à 3 facteurs contrôlés (sexe des animaux, bouf ou vache), moment de l'abattage ( 2 à 8 h ou 8 à $\mathrm{I} 3 \mathrm{~h}$ après le repas), régime alimentaire, et 2 covariables, poids du contenu de rumen et teneur en matière sèche de ce contenu.

Le moment de l'abattage, le poids vif de l'animal ou son sexe n'ont pas eu d'effet significatif. Cependant, comme l'amplitude de variation du poids vif des animaux était relativement faible à l'intérieur de chaque classe d'aliment, on a poursuivi l'analyse en exprimant le poids des contenus en pourcentage du poids vif afin de pouvoir appliquer nos relations à une gamme de poids vif plus étendue.

I,e poids du contenu du reste du tube digestif est étroitement lié au poids du contenu du rumen : $r=0,65$ (fig. I et équation $\mathbf{I}$ du tabl. 2 ). Par conséquent, le poids du contenu total peut être estimé convenablement à partir de celui du contenu du rumen par l'équation (2) dont l'écart-type résiduel $\left(S_{d}=0,89\right)$ correspond à $6 \mathrm{p}$. Ioo du poids du contenu total. L'erreur d'estimation est inférieure à I p. I oo du poids vif.

La connaissance de la teneur en matière sèche du contenu du rumen améliore de très peu la précision de l'estimation $\left(S_{d}=0,86\right)$. En revanche, lorsqu'on distingue les différents régimes alimentaires, la relation devient plus étroite $\left(r=0,82 S_{\boldsymbol{d}}=0,69\right.$, soit $4,7 \mathrm{p}$. I00 du contenu total) et fait apparaître un coeffi- 

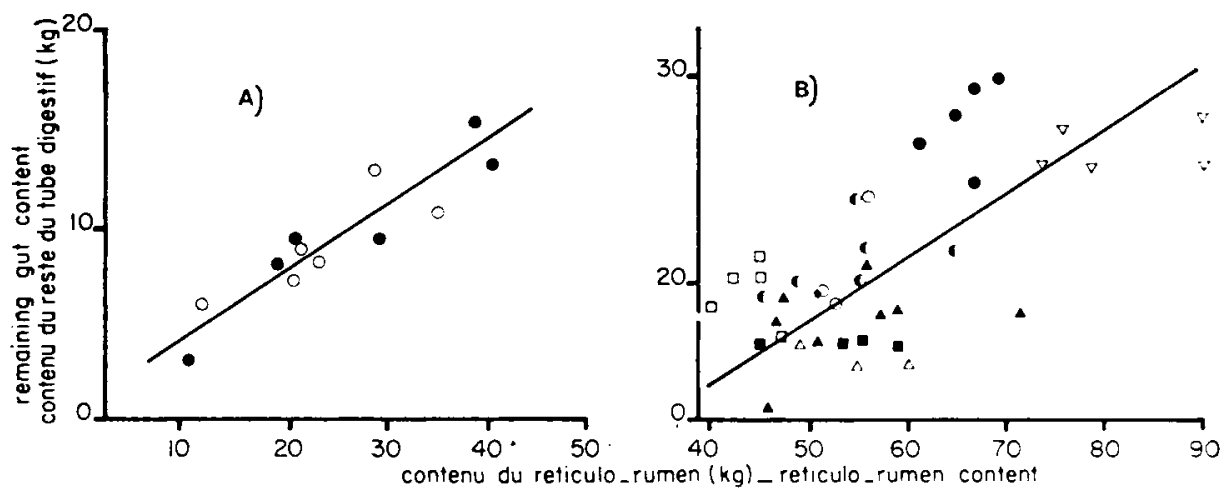

FIG. I. - Relation entre le poids du contenu du réticulo-rumen et le poids $d u$ contenu $d u$ reste $d u$ tube digestif des bovins.

Relationship between the reticulo-rumen content and the remaining gut content.

A) Variations selon le poids des animaux ( 120 à $650 \mathrm{~kg}$ ) chez des taurillons Pie Noirs (o) et Charolais $(\bullet)$ recevant un régime comportant 80 p.roo de concentré et 20 p. Ioo de fourrage. Variations with the weight of animals (120 to $650 \mathrm{~kg}$ ) in Friesian bulls (0) and Charolais ones (•) fed a diet containing $80 \mathrm{p}$. roo concentrate and $z 0 \mathrm{p}$. too roughage.

$B$ ) Variations chez des bœufs et des vaches adultes selon le régime alimentaire :

Variations in steers and adult cows with the feeding diet:

o Foin de luzerne - Lucerne hay.

- Foin de luzerne et betteraves - Lucerne hay and fodder beets.

- Foin et orge - Hay and barley.

$\triangle$ Choux - Cabbages.

$\checkmark$ Foin de graminées - Grass hay.

- Ration agglomérée - Pelleted diet.

- Concentré et foin - Concentrate and hay.

- Herbe - Fresh herbage.

\section{TABIEAU 2}

Relation entre le poids total (Y) du contenu digestif et le poids du contenu du rumen (X) (en p. тоo du poids vif)

Relationship between weight of the total digestive tract content and weight of the rumen content ( $p$. Ioo of livewcight)

(I)

(2)

(3)

(4)

(5)

$$
\begin{aligned}
(\mathrm{Y} \cdots \mathrm{X}) & =\mathrm{I}, 05+0,28 \mathrm{X} \\
\mathrm{Y} & =\mathrm{I}, 05+\mathrm{I}, 28 \mathrm{X} \\
\mathrm{Y} & =2,39+\mathrm{I}, 15 \mathrm{X}+\mathrm{A}{\left({ }^{*}\right)} \\
\mathrm{A} & =4,28-6,2 \mathrm{dMO}\left(^{* *}\right) \\
\mathrm{Y} & =6,67+\mathrm{I}, \mathrm{1} 5 \mathrm{X}--6,2 \text { dMO }\left(^{* *}\right)
\end{aligned}
$$

$$
\begin{aligned}
& \mathbf{S}_{\mathbf{d}}=0,89 \\
& \mathbf{S}_{\mathbf{d}}=0,89 \\
& \mathbf{S}_{\mathbf{d}}=0,69 \\
& \mathbf{S}_{\mathbf{d}}=0,38 \\
& \mathbf{S}_{\mathbf{d}}=0,79
\end{aligned}
$$

${ }^{*}$ ) A varie de - I,I à $+\mathrm{I,2}$ selon la nature du régime (figure 2 ).

A varies from - I.I to $+\mathbf{I} .2$ according to the nature of the diet (fig. 2).

(**) dMO : digestibilité de la matière organique de la ration (dMO $<\mathrm{I}$ ).

Digestibility of the organic matter of the diet $($ dMO $<\mathrm{I})$. 
cient A significativement différent de zéro (équation 3 du tabl. 2). Dans le cas des régimes étudiés, le coefficient $A$ a varié entre $+I, 2$ et $-I, I$ selon la nature de la ration (fig. 2). Sa valeur est assez étroitement liée $(r=0,87)$ à la digestibilité de la matière organique de la ration (dMO) (tabl. 2, équation 4, fig. 2).

Dans la pratique, il est assez difficile de donner une valeur très précise à ce coefficient A si on ne connaît que la nature du régime. Il varie en effet de $-0,5$ à - I I I pour les fourrages verts très digestibles et les rations riches en concentrés, et de $+0,5$ à $+\mathbf{I}, 2$ pour les foins. La précision de l'estimation obtenue à partir de l'équation (2) est donc inférieure à l'écart-type résiduel $\left(S_{d}=0,69\right)$.

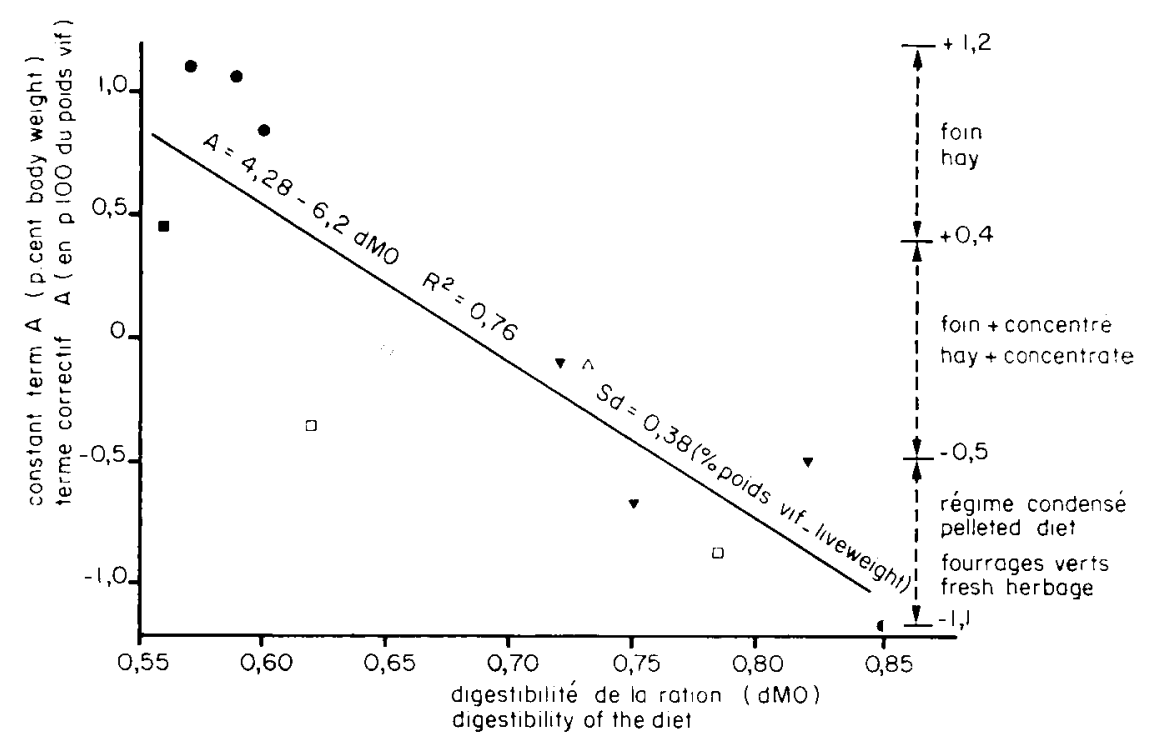

FIs. 2. - I'anation selon la digestibilite de la ration, du terme constant A dans la relation entre le contenu du véticulo-rumen (CRU) et celui du reste du tube digestif (CRL) : CRL ( $p$. Ioo poids $v i f)=2,39+A+0,15(R V$ (p. 100 poids vif).

rariation, with the digestibility of the diet, of the constant term $A$ of the velation betwien the reticulorumen content (CRU) and the remaining gut content (CRE): CRE ( $p$. Ioo liveweight) =. $2,39+A+o, 15$ CRU (p. Ioo livereight).

Lorsqu'on connait, ou que l'on peut estimer la digestibilité de la matière organique de la ration distribuée aux animaux, le poids du contenu digestif total peut donc être déterminé par l'équation (5) du tableau 2 dont l'écart-type résiduel $(0,79)$ est égal à 5,4 p. Ioo du contenu total.

Nous avons vérifié sur quelques exemples que ces relations obtenues essentiellement à partır d'animaux adultes et de bœufs de plus de $450 \mathrm{~kg}$ s'appliquaient aux jeunes bovins en croissance (fig. I : taurillons Charolais et Pie Noirs de Izo à $650 \mathrm{~kg})$.

\section{Conclusions}

Ces résultats peuvent permettre aux expérimentateurs, capables de peser le contenu du rumen à l'abattage ou après vidange à travers une canule, de connaître le poids total du contenu digestif à 5 p. roo près et le poids vif vide de 
1'animal à I p. Ioo près. On utilise l'équation (2) si seul le poids du contenu du rumen est connu, et les équations (3) ou (5) si la nature de la ration ou sa digestibilité sont connues.

Toutefois, les animaux n'ont pas été abattus plus de I3 heures après leur repas; dans les abattoirs, les animaux subissent un jeûne plus prolongé et les équations précédentes ne peuvent peut-être pas leur être appliquées.

Accepté pour publication en juillet 1978 .

\section{Summary}

\section{Estimation of the weight of digesta in cattle from the weight of the rumen content}

From data obtained in 198 slaughtered cattle, the total weight of digesta was estimated from the weight of the reticulo-ruminal content.

The accuracy of this estimation was about $5 \mathrm{p}$. 100 (see equation 2, Table 2 ). Thus, empty body weight could be determined from liveweight with a deviation lower than I p. Ioo. The accuracy was higher when digestibility of the diet was known. 